\title{
A New Comet (the Great Comet of Sept. 1882).
}

A brilliant comet first seen in Australia on the morning of the $9^{\text {th }}$ of September*) has since been observed here on every occasion, when the very cloudy weather which has prevailed would permit.

It became so bright just before perihelion that it was seen at noon with the naked eye, within four degrees of the sun on the $I 7^{\text {th }}$ and was observed on the meridian with the transit circle on three days. It is now (Sept. $25^{\text {th }}$ ) again visible in the early morning. On the $24^{\text {th }}$ it was easily seen with a $4 \frac{1}{2}$ " telescope three hours after sunrise. Its tail was seen $15^{\circ}$ long in the bright dawn and was about $I^{\circ}$ wide at the end. This morning although markedly less bright it was seen in the same telescope 20 minutes after sunrise.

From observations obtained on the $9^{\text {th }}, 13^{\text {th }}$ and $16^{\text {th }} \mathrm{Mr}$. E. J. White, chief assistant, computed a first approxiion to an orbit which is here given:

$$
\begin{gathered}
T=\text { Sept. 1 7,1 } 75 \\
x=275^{\circ} \mathbf{1 2}^{\prime} \\
8=3533^{8} \\
i=38 \text { 1 } \\
\log q=7.6906 \\
\text { Motion retrograde. }
\end{gathered}
$$

The exceedingly small perihelion distance, as well as the other elements of this comet exhibit a decided similarity to that of 1843 .

The positions hitherto obtained are attached.

Places of the Great Comet of September 1882 from observations made with the North Equatorial, aperture $4 \frac{1}{2}$ inches, and the transit circle of the Melbourne Observatory.

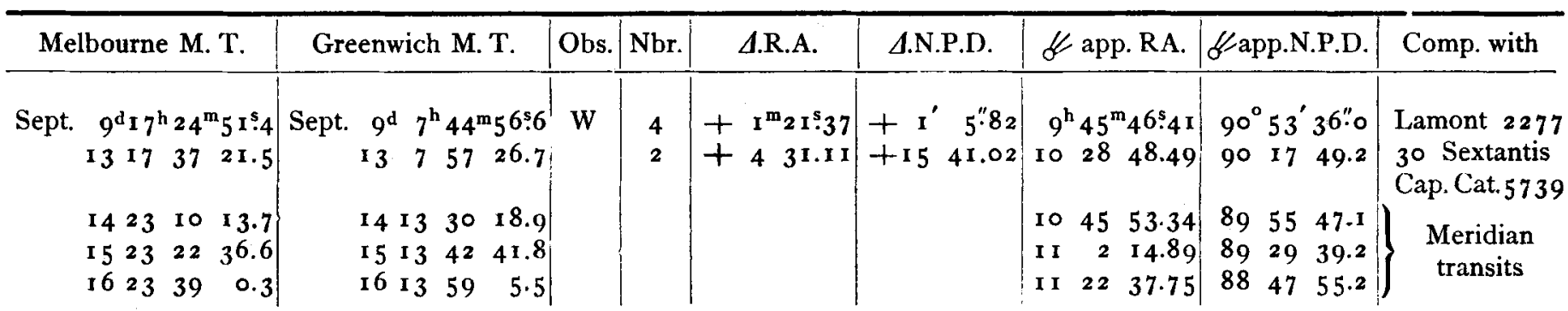

The meridian transit of Sept. 15 was observed with great difficulty the comet being obscured by cloud.

Melbourne Observatory 1882 Sept. 25.

Rob. F. Ellery.

*) Nach dem Science Observer Circular 28 hat Dr. Gould in Cordoba den Cometen am 6. Sept. entdeckt. Kr.

\section{Ueber den grossen Septembercometen 1882.}

Auszug aus einem Schreiben von Herrn $C h$. André, Director der Sternwarte zu Lyon, an den Herausgeber.

Le 24. Septembre dernier la tête de la comète était limitée par une ligne brillante très nette et son noyau était sensiblement circulaire.

Le Io. Octobre de grands changements s'étaient produits - la ligne brillante, qui limitait la tête a disparu, le noyau s'est allongé et a les dimensions suivantes:

$$
\begin{aligned}
& \times 5^{\prime \prime} \text { en largeur, } \\
& 49^{\prime \prime} \text { en longueur, } \\
& 281^{\circ} \text { comme angle de position. }
\end{aligned}
$$

De plus ce noyau présente à son intérieur deux centres de condensation distincts, le premier plus brillant très près de l'extrémité antérieure de la tête, le second moins intense et au deux tiers environ du noyau, à partir de cette extrémité antérieure.

Le I 2. Octobre le noyau présente semblablement les mêmes apparences. Mais la queue est partagée en deux parties presque égales par une nervure médiane et limitée sur chaque bord par deux arêtes rectilignes; la moitié nord est peu brillante, l'autre l'est rélativement beaucoup; les deux bords se prolongent faibles un peu au dessous du noyau mais sans se rejoindre.

La longueur de la queue était de $17^{\circ}$.

Observatoire de Lyon 1882 Octobre 18.

Ch. André. 